\title{
Effect of paclitaxel-coated balloon angioplasty on side branch lesion and cardiovascular outcomes in patients with de novo true coronary bifurcation lesions undergoing percutaneous coronary intervention
}

\section{Youmei Li}

\author{
Xinqiao Hospital
}

Qi Mao ( $\square$ holygeneral1214@163.com )

Army Medical University https://orcid.org/0000-0002-6040-0116

\section{Huanyun Liu}

First People's Hospital of Chongqing Liang Jiang New Area: The First People's Hospital of Chongqing Liangjiang New Area

Denglu Zhou

Xinqiao Hospital

Jianhua Zhao

Xinqiao Hospital

\section{Research Article}

Keywords: paclitaxal-coated balloon, percutaneous coronary intervention, bifurcation lesion, late lumen loss, cardiovascular events

Posted Date: April 12th, 2021

DOl: https://doi.org/10.21203/rs.3.rs-391987/v1

License: (c) (1) This work is licensed under a Creative Commons Attribution 4.0 International License. Read Full License

Version of Record: A version of this preprint was published at Cardiovascular Drugs and Therapy on July 9th, 2021. See the published version at https://doi.org/10.1007/s10557-021-07225-8. 


\section{Abstract \\ Purpose}

To compare the effects of paclitaxal-coated balloon (PCB) versus conventional balloon (CB) on side branch (SB) lesion and cardiovascular outcomes in patients with de novo true bifurcation lesions.

\section{Methods}

In total, 219 patients with de novo true bifurcation lesions were enrolled and divided into PCB group (102 cases) and CB group (117 cases) according to angioplasty strategy in SB. Drug-eluting stent (DES) was implanted in main vessel (MV) for each subject. All subjects underwent a 12-month follow-up for late lumen loss (LLL), restenosis and major adverse cardiovascular events (MACE) after percutaneous coronary intervention (PCI). MACEs included cardiac death, nonfatal myocardial infarction and angina pectoris.

\section{Results}

There were no differences in diameter, minimum lumen diameter (MLD) and stenosis for bifurcation lesions between the two groups before and immediately after $\mathrm{PCl}(\mathrm{P}>0.05)$. After 12-month follow-up, no differences occurred in MV-MLD and MV-LLL between the two groups $(P>0.05)$; SB-MLD in PCB group was higher than that in $\mathrm{CB}$ group $(1.97 \pm 0.36 \mathrm{~mm}$ vs. $1.80 \pm 0.43 \mathrm{~mm}, \mathrm{P}=0.007)$; $S B-L L L$ in $P C B$ group was lower than that in $\mathrm{CB}$ group $(0.11 \pm 0.18 \mathrm{~mm}$ vs. $0.19 \pm 0.25 \mathrm{~mm}, \mathrm{P}=0.024)$. Multivariate $\mathrm{COX}$ analyses indicated that $\mathrm{PCB}$ group had lower MACE risk than $\mathrm{CB}$ group $(\mathrm{HR}=0.480,95 \% \mathrm{Cl} 0.244-0.941$, $P=0.033)$.

\section{Conclusion}

PCB could decrease SB-LLL and MACE risk in patients with de novo true coronary bifurcation lesion 12 months after single-DES intervention.

\section{Introduction}

Interventional therapy for coronary bifurcation diseases accounts for about $15 \%-20 \%$ of percutaneous coronary intervention (PCI) [1]. Coronary bifurcation lesions are complicated and diverse, difficult to intervene with high ischemic risk [2,3]. In the drug-eluting stent (DES) era, although the single-stent intervention strategy can reduce the risks of main vessel (MV) restenosis and target vessel revascularization, late lumen loss (LLL) of side branch (SB) has not significantly relieved as expected [4, 5]. Therefore, the interventional treatment of bifurcation lesions is still full of challenges. 
Drug-coated balloon (DCB) locally releases anti-cell proliferation drugs to lesion intima through delivery and dilation to inhibit restenosis [6]. In the era of stentless PCI, DCB could be considered as an interventional option for small-vessel lesion, in-stent restenosis (ISR), and bifurcation lesion [7]. However, few studies have been reported in de novo bifurcation lesion, especially lack of evidence for SB intervention based on the patients with true bifurcation lesions. Previous evidences showed that paclitaxel-coated balloons (PCB) significantly reduced ISR risk $[8,9]$. Here, we hypothesize that PCB angioplasty may also decrease SB-LLL and the incidence of adverse cardiovascular events in the singlestent intervention strategy for bifurcation lesions. Therefore, this study aims to compare the effects of PCB versus conventional balloon (CB) on SB lesion and cardiovascular outcomes in patients with de novo true bifurcation lesions.

\section{Methods}

\section{Study Design and Population}

This was a retrospective cohort study, adhering to the Strengthening the Reporting of Observational Studies in Epidemiology statement. The study protocol complied with the Declaration of Helsinki and was approved by Xinqiao hospital ethics committee, Army Medical University (Chongqing, China).

We evaluated 413 patients with true bifurcation lesion undergoing $\mathrm{PCl}$ who admitted to cardiology department between September 2016 and March 2019 in our hospital. The inclusion criteria were as follows: (1) de novo bifurcation disease, lumen stenosis $\geq 50 \%$; (2) age $\geq 18$ years old; (3) true coronary bifurcation lesions (Medina classification 1.1.1, 1.0.1, 0.1.1); (4) second-generation drug-eluting stent (DES) into the main vessel (MV) and balloon angioplasty in side branch (SB); (5) residual stenosis $<50 \%$ after pre-dilation. The exclusive criteria were as follows: (1) previous $\mathrm{PCl}$; (2) previous coronary artery bypass grafting (CABG); (3) acute myocardial infarction; (4) stenting in SB; (5) contraindications for dual antiplatelet therapy; (6) malignant disease; (7) arterial dissection; (8) severe calcified lesions that cannot be successfully dilated; (9) primary kidney diseases (including primary nephritis syndrome, primary nephrotic syndrome, interstitial nephropathy and hereditary nephropathy), severe hepatic dysfunction, bleeding and coagulation diseases. In our hospital, CABG was the only way of revascularization for unprotected left main disease (UPLM), so UPLM and its bifurcation were included in CABG. Finally, a cohort of 219 patients was enrolled in this study. According to balloon angioplasty strategy in SB, the subjects were divided into conventional balloon (CB) group (117 cases) and paclitaxel-coated balloon (PCB) group (102 cases).

\section{PCI Procedure}

Everolimus-coated DES (Promus Premier, Boston Scientific, Marlborough, USA) was implanted in MV. CB (Quantum Maverick, Boston Scientific, Marlborough, USA) or PCB (SeQuent Please, Braun Melsungen, Berlin, Germany) was used for SB angioplasty. Hydrophilic coating and lipophilic paclitaxel were evenly distributed on the balloon surface that made paclitaxel easier to penetrate from balloon surface to vascular intima [10]. After pre-dilation for MV and SB, if no dissection or type A/B dissection existed in SB 
and residual stenosis of SB was less than $30 \%$ with TIMI grade 3 blood flow; DES was firstly implanted into MV with guide wire or balloon for SB protection; guide wire passed the stent mesh to exchange; then balloon was utilized to dilate the ostial lesion and SB, respectively; the standard balloon to artery ratio (0.8-1: 1) was mandated in procedure. If pre-dilation was not successfully (C-F type dissection or TIMI grade 0 or 1 blood flow in SB), double-stent interventional strategy was directly performed (Culotte or Crush could be considered by means of the characteristics of bifurcation lesion).

\section{Definition}

Coronary bifurcation disease was the lesion with severe stenosis in the MV and SB, respectively or together [11]. True bifurcation lesions referred to the ones in which both the MV and SB were affected and corresponded to Medina classification 1.1.1, 1.0.1, and 0.1.1 [12]. Medina classification was based on the order of the proximal/distal MV and SB, and was displayed as with (1) or without (0) lesion [13]. Unprotected left main was defined as the absence of protective vessels that no collateral circulation was established or no CABG grafts [14]. Restenosis was defined as diameter stenosis $\geq 50 \%$ at follow-up. Late lumen loss (LLL) referred to the difference in MLD between stenting and follow-up [15]. Acute myocardial infarction refer to the fourth universal definition, when troponin value exceeds the 99th percentile upper reference limit and combines at least one of following characteristics: (1) symptoms of myocardial ischemia; (2) new changes in ischemic electrocardiogram or emerging pathological Q waves; (3) imaging evidence of new loss of viable myocardium or new regional wall motion abnormality [16]. Angina pectoris was defined as ischemic chest pain that drived readmission, based on symptom, electrocardiogram characteristics and troponin.

\section{Data Collection and Follow-up}

Clinical data were collected from medical records by trained physicians including demographic data, medical history, laboratory indicators and essential drug therapy. Coronary angiography data were independently assessed by two interventional cardiologists including bifurcation site, Medina classification, diameters, minimum lumen diameter (MLD) and lumen stenosis, and the two interventional cardiologists were blind as to treatment. All angiography was performed under the same standard conditions, and quantitative coronary analysis (QCA) was performed using the QAngio XA system version 7.3.74.0 (Medis Medical Imaging Systems, Leiden, Nertherlands) [17].

Primary outcomes and bifurcation lesions were included in the follow-up process. Primary outcomes were major adverse cardiovascular events (MACE) defined as the composite of cardiac death, nonfatal myocardial infarction and angina pectoris. Reassessment of bifurcation lesions involved MLD, LLL and restenosis for MV and SB, respectively. All patients had a 12-month follow-up for angiography and cardiovascular events after $\mathrm{PCl}$, and related data were obtained from hospital records and by interviewing patients and their families.

\section{Statistical Analysis}


PASS software version 11.0 was used to calculate power and determined the sample size based on previous studies and our estimates [18, 19]. Calculation formula for sample size as follow: see formula 1 in the supplementary files.

Finally, each group needed 98 cases at least.

SPSS software version 24.0 (SPSS, Inc, Chicago, Illinois) was utilized for statistical analyses. Continuous variables were expressed as mean $\pm S D$ and categorical variables were expressed as frequencies with percentages. The $t$ test was used to compare continuous data and differences in categorical data were assessed by the Chi-squared test or Fisher exact test (two-sided). Cox regression analyses were performed to evaluate the association of PCB angioplasty with SB restenosis and MACE. Multivariate Cox models were adjusted for established cardiovascular risk factors (age, male, hypertension, diabetes, smoking, hyperlipidemia, bifurcation site and Medina classification). P values of less than 0.05 were considered statistically significant.

\section{Results}

\section{Characteristics of Study Population}

A total of 219 patients with de novo true coronary bifurcation lesions were involved in this study including 117cases in CB group and 102 cases in PCB group, and no patients were lost to follow-up. No adverse cardiovascular events occurred during $\mathrm{PCl}$ procedure such as malignant arrhythmia, cardiac death, salvage $\mathrm{PCl}$ and acute $\mathrm{CABG}$. Blood flow immediately after $\mathrm{PCl}$ was TIMI grade 3 . No differences appeared in clinical characteristics between the two groups $(P>0.05)$ (Table 1). There were no significant differences in diameter, MLD and stenosis between the two groups before and immediately after $\mathrm{PCI}$ ( $P>0.05$ ) (Table 1). In this study, PCB group had more left main bifurcation lesions (58.82\% vs. $39.32 \%)$ and more Medina 1.1 .1 lesions (26.47\% vs. $17.95 \%$ ) compared with CB group (Table 1).

\section{Reassessment of Bifurcation Lesions}

All patients underwent coronary angiography review at 12-month follow-up after $\mathrm{PCl}$ to assess the severity of coronary bifurcation lesions. At the follow-up point, there was no significant differences in MVMLD and MV-LLL between the two groups ( $P>0.05)$; SB-MLD in PCB group was higher than that in $C B$ group $(1.97 \pm 0.36 \mathrm{~mm}$ vs. $1.80 \pm 0.43 \mathrm{~mm}, \mathrm{P}=0.007)$; $S B-L L L$ in $\mathrm{PCB}$ group was lower than that in $\mathrm{CB}$ group $(0.11 \pm 0.18 \mathrm{~mm}$ vs. $0.19 \pm 0.25 \mathrm{~mm}, \mathrm{P}=0.024)$ (Table 2 ).

\section{Clinical Outcomes}

Cox proportional risk regression model was performed to evaluate the association of PCB angioplasty with MACE and component events. PCB group had 5 cases (4.90\%) in SB restenosis and 13 cases (12.75\%) in MACE; while CB group had 9 cases $(7.69 \%)$ in SB restenosis and 28 cases $(23.93 \%)$ in MACE. There was no significant difference in restenosis $(\mathrm{HR}=0.620,95 \% \mathrm{Cl} 0.208-1.850, \mathrm{P}=0.383)$ between the two groups, but PCB group had lower MACE risk than CB group ( $\mathrm{HR}=0.489,95 \% \mathrm{Cl} 0.253-0.945, \mathrm{P}=0.028)$. 
After adjustment for possible compounding factors (including clinical parameters and bifurcation characteristics), multivariate analyses indicated that PCB group still had lower MACE risk than CB group $(\mathrm{HR}=0.480,95 \% \mathrm{Cl} 0.244-0.941, \mathrm{P}=0.033)$, the increased MACE risk was mainly attributed to more angina in $\mathrm{CB}$ group $(\mathrm{HR}=0.515,95 \% \mathrm{Cl} 0.247-1.076, \mathrm{P}=0.078)$ (Table 3).

\section{Discussion}

$\mathrm{PCl}$ for coronary bifurcation lesions was challenging. Compared with simple lesions, bifurcation lesions had more severe plaque burden and was more vulnerable to erosion and rupture [20]. Even if PCI procedure was gradually improving, displacement of crest and plaque, plaque rupture, and SB ostium elastic retraction still often occurred during PCl for bifurcation lesions [21]. In single-stent intervention, even if "wire jailed" or "balloon jailed" was utilized, severe stenosis or occlusion of SB often occurred and the risk of long-term restenosis was still high [22]. In double-stent strategy, there were still also many shortcomings such as complicated procedure, long duration and high radiation exposure; furthermore multiple stent trabeculars and polymers mediated intimal proliferation and delayed endothelialization, thereby increasing the risks of in-stent restenosis (ISR) and delayed stent thrombosis that required longer dual antiplatelet therapy and brought higher bleeding risk and financial burden to patients [23].

Stenting in MV combined with angioplasty for SB was a common interventional procedure for bifurcation lesions, but long-term follow-up showed that severe stenosis tended to occur in SB [24]. DK-CRUSH study demonstrated that stenting in MV combined with angioplasty for SB or stenting in SB could not prevent long-term restenosis in SB [25]. DCB released cytotoxic agent to inhibit intimal proliferation, thus alleviating restenosis. Lots of evidences indicated that DCB could replace DES for ISR treatment and similar findings had also been reported in the intervention of de novo lesions [26]. However, few studies had explored DCB intervention, especially PCB intervention, on de novo true bifurcation lesions. Current evidence still mainly focused on the effect of DCB intervention on de novo lesions and its ISR.

In bifurcation studies, Lee et al. [27] reported 114 patients with ISR of LM bifurcation lesions were assigned to DES group and DCB group according to SB intervention strategy; the one-year follow-up results showed that DCB group had lower cardiogenic mortality than DES group ( $0 \%$ vs. $10.7 \%)$. Harada et al. [28] investigated the effects of different PCBs on ISR of bifurcation lesions, and the results showed no significant difference. Most DCB-related studies based on bifurcation lesions had small sample sizes and no adequate control group. In de novo lesion studies, DEBSIDE study and PEPCAD $V$ study showed DCB safe and effective for de novo bifurcation lesions, and the risks of ISR and target lesion revascularization decreased significantly $[29,30]$. However, considering no CB control group in the above studies, the external validity of the results was low and required for further verification. The DEBIUT study compared the effects of $D C B+$ bare metal stent (BMS), $C B+B M S$, and $C B+D E S$ on the cardiovascular outcomes in patients with bifurcation lesions. The results indicated that $D C B+B M S$ group did not exceed the other two groups in the risks of SB restenosis and MACE [31]. BABILON study also explored the effects of $D C B+B M S$ versus $C B+D E S$ on the prognosis of de novo bifurcation lesions, and it found that no significant difference occurred between the two groups in SB-LLL [32]. Although both DEBIUT study and 
BABILON study revealed that DCB was not superior to CB for de novo bifurcation lesions; BMS was implanted in MV for DCB group while DES was implanted in MV for control group in both studies. As known, since BMS could not effectively reduce the risks of long-term restenosis and MACE compared with DES, such grouping might offset the potential benefits of DCB.

Our study compared the effects of $P C B+D E S$ versus $C B+D E S$ on the efficacy for de novo true bifurcation lesions. The results indicated that PCB increased SB-MLD, deceased SB-LLL and the incidence of MACE significantly after 12-month follow-up compared with $\mathrm{CB}$. Although statistical analysis revealed no difference between the two groups in risk of restenosis, given short follow-up period and high standard definition of restenosis, the results were still interpretable and were required to be fully verified in the future. The effect of PCB on reducing MACE risk could be attributed to the following points: (1) PCB effectively lowered SB-LLL to ameliorate myocardial perfusion, thereby decreased the risk of angina or ischemia-driven revascularization [33]. (2) Through balloon carrier, paclitaxel exerted anti-cell proliferation effect to strongly inhibit the occlusion of SB ostium, thus avoiding SB loss [34]. (3) Successful pretreatment for target lesion before PCB angioplasty could help improve blood perfusion [35]. Our study still had several limitations. Firstly, this was a single center study with potential bias. Secondly, our sample size was small and follow-up duration was short. Thirdly, PCl was not completed by the same interventional cardiologist for each subject. In view of the complexity of bifurcation lesions and the difficulty of intervention, $\mathrm{PCl}$ procedure might affect cardiovascular outcomes. Finally, our study population was of Chinese origin.

\section{Conclusion}

Our study revealed that PCB could decrease SB-LLL and MACE risk in patients with de novo true coronary bifurcation lesions 12 months after single-DES intervention. For true bifurcation lesions, after sufficient pre-dilation, PCB angioplasty in SB combined with DES stenting in MV can effectively reduce the cardiovascular events, which can be performed as reliable option for intervention. Given the limitations of current study, further multicenter, large-scale, long follow-up duration studies are required to validate the implication of these results.

\section{Declarations}

\section{Author Contributions}

Qi Mao contributed to the study conception and design. Youmei Li and Huanyun Liu analyzed the patient data regarding coronary angiograph. Data collection was performed by Denglu Zhou and Jianhua Zhao. The first draft of this manuscript was written by Youmei Li and Qi Mao. All authors commented previous versions of the manuscript. All authors read and approved the final manuscript.

\section{Funding Information}

This study was funded by the Natural Science Foundation of Chongqing (grant no. cstc2017jcyjAX0042). 


\section{Compliance with Ethical Standards}

\section{Conflicts of Interest}

The authors have no conflicts of interest to declare.

\section{Ethics Approval}

All procedures performed in this study involving human participants were in accordance with the ethical standards of the institution (ethics committee of Xinqiao hospital, Chongqing). Informed consent was obtained from all individual participants of this study.

\section{Data Availability}

The data used to support the findings of this study are available from the corresponding author upon request.

\section{References}

1. Lassen J F, Holm N R, Banning A, et al. Percutaneous coronary intervention for coronary bifurcation disease: 11th consensus document from the European Bifurcation Club. Eurolntervention. 2016; 12: 38-46.

2. Généreux $P$, Kumsars I, Lesiak $M$, et al. A randomized trial of a dedicated bifurcation stent versus provisional stenting in the treatment of coronary bifurcation lesions. J Am Coll Cardiol. 2015; 65: 533-43.

3. Al Suwaidi J, Berger P B, Rihal C S, et al. Immediate and long-term outcome of intracoronary stent implantation for true bifurcation lesions. J Am Coll Cardiol. 2000; 35: 929-36.

4. Kumsars I, Holm N R, Niemelä M, et al. Randomised comparison of provisional side branch stenting versus a two-stent strategy for treatment of true coronary bifurcation lesions involving a large side branch: the Nordic-Baltic Bifurcation Study IV. Open Heart. 2020; 7: e947.

5. Zamani $P$ and Kinlay S. Long-term risk of clinical events from stenting side branches of coronary bifurcation lesions with drug-eluting and bare-metal stents: an observational meta-analysis. Catheter Cardiovasc Interv. 2011; 77: 202-12.

6. Yerasi C, Case B C, Forrestal B J, et al. Drug-coated balloon for de novo coronary artery disease. J Am Coll Cardiol. 2020; 75: 1061-73.

7. Rissanen T T, Uskela S, Eränen J, et al. Drug-coated balloon for treatment of de-novo coronary artery lesions in patients with high bleeding risk (DEBUT): a single-blind, randomised, non-inferiority trial. Lancet. 2019; 394: 230-9.

8. Byrne R A, Joner M, Alfonso F and Kastrati A. Drug-coated balloon therapy in coronary and peripheral artery disease. Nat Rev Cardiol. 2014; 11: 13-23. 
9. Ang H, Lin J, Huang Y Y et al. Drug-coated balloons: technologies and clinical applications. Curr Pharm Des. 2018;24:381-96.

10. Vos N S, Fagel N D, Amoroso G, et al. Paclitaxel-coated balloon angioplasty versus drug-eluting stent in acute myocardial infarction. JACC: Cardiovasc Interv. 2019; 12: 1691-9.

11. Elwany $M$, Palma G D and Cortese B. Treatment of coronary bifurcation lesions: current knowledge and future perspectives. Future Cardiol. 2018; 14: 165-79.

12. Leesar M A, Hakeem A, Azarnoush K and Thuesen L. Coronary bifurcation lesions: Present status and future perspectives. Int J Cardiol. 2015; 187: 48-57.

13. Perl L, Witberg G, Greenberg G, Vaknin-Assa H, Kornowski R and Assali A. Prognostic significance of the Medina classification in bifurcation lesion percutaneous coronary intervention with secondgeneration drug-eluting stents. Heart Vessels. 2020; 35: 331-9.

14. Collet $C$, Capodanno D, Onuma $Y$, et al. Left main coronary artery disease: pathophysiology, diagnosis, and treatment. Nat Rev Cardiol. 2018; 15: 321-31.

15. Asano T, Serruys $P$ W, Collet $C$, et al. Angiographic late lumen loss revisited: impact on long-term target lesion revascularization. Eur Heart J. 2018; 39: 3381-9.

16. Thygesen K, Alpert J S, Jaffe A S, et al. Fourth universal definition of myocardial infarction (2018)[J]. Circulation. 2018; 138: e618-e651.

17. Sheth T, Pinilla-Echeverri N, Moreno R, et al. Nonculprit lesion severity and outcome of revascularization in patients with STEMI and multivessel coronary disease[J]. J Am Coll Cardiol. 2020; 76:1277-1286.

18. Yerasi C, Case B C, Forrestal B J, et al. Drug-coated balloon for de novo coronary artery disease[J]. J Am Coll Cardiol. 2020; 75: 1061-1073.

19. Zheng W, Li Y, Tian J, et al. Effects of ticagrelor versus clopidogrel in patients with coronary bifurcation lesions undergoing percutaneous coronary intervention[J]. BioMed Res Int. 2019; 2019: 112.

20. Zhang J, Ye F, Xu K, et al. Multicentre, randomized comparison of two-stent and provisional stenting techniques in patients with complex coronary bifurcation lesions: the DEFINITION II trial. Eur Heart J. 2020; 41: 2523-36.

21. Naganuma T, Chieffo A, Meliga E, et al. Long-term clinical outcomes after percutaneous coronary intervention for ostial/mid-shaft lesions versus distal bifurcation lesions in unprotected left main coronary artery: the DELTA Registry (drug-eluting stent for left main coronary artery disease): a multicenter registry evaluating percutaneous coronary intervention versus coronary artery bypass grafting for left main treatment. JACC Cardiovasc Interv. 2013; 6: 1242-9.

22. Ford T J, McCartney P, Corcoran D, et al. Single- versus 2-stent strategies for coronary bifurcation lesions: a systematic review and meta-analysis of randomized trials with long-term follow-up. J Am Heart Assoc. 2018; 7(11): e008730.

23. Chen S, Zhang J, Han Y, et al. Double kissing crush versus provisional stenting for left main distal bifurcation lesions: DKCRUSH-V randomized trial. J Am Coll Cardiol. 2017; 70: 2605-17. 
24. Burzotta F, Trani C, Todaro D, et al. Prospective randomized comparison of sirolimus- or everolimuseluting stent to treat bifurcated lesions by provisional approach. JACC: Cardiovasc Interv. 2011; 4: 327-35.

25. Chen S, Santoso T, Zhang J, et al. Clinical outcome of double kissing crush versus provisional stenting of coronary artery bifurcation lesions. Circ: Cardiovasc Interv. 2017; 10(2): e004497.

26. Tang Y, Qiao S, Su X, et al. Drug-coated balloon versus drug-eluting stent for small-vessel disease. JACC: Cardiovasc Interv. 2018; 11: 2381-92.

27. Lee W, Hsueh S, Chen C, et al. The comparison of clinical outcomes after drug-eluting balloon and drug-eluting stent use for left main bifurcation in-stent restenosis. Int Heart J. 2018; 59: 935-40.

28. Harada Y, Colleran R, Pinieck S, et al. Angiographic and clinical outcomes of patients treated with drug-coated balloon angioplasty for in-stent restenosis after coronary bifurcation stenting with a twostent technique. Eurolntervention. 2017; 12: 2132-9.

29. Berland J, Lefevre T, Brenot P, et al. DANUBIO-a new drug-eluting balloon for the treatment of side branches in bifurcation lesions: six-month angiographic follow-up results of the DEBSIDE trial. Eurolntervention. 2015; 11: 868-76.

30. Mathey D G, Wendig I, Boxberger M, Bonaventura K and Kleber F X. Treatment of bifurcation lesions with a drug-eluting balloon: the PEPCAD V (Paclitaxel Eluting PTCA Balloon in Coronary Artery Disease) trial. Eurolntervention. 2011; 7 Suppl K: K61-5.

31. Belkacemi A, Agostoni P, Voskuil M and Stella P R. Coronary bifurcation lesions treated with the drugeluting balloon: a preliminary insight from the DEBIUT study. Eurolntervention. 2011; 7 Suppl K: K669.

32. Lopez M J, Nogales A J, Doncel V L, et al. A prospective randomised study of the paclitaxel-coated balloon catheter in bifurcated coronary lesions (BABILON trial): 24-month clinical and angiographic results. Eurolntervention. 2014; 10: 50-7.

33. Vos N S, Fagel N D, Amoroso G, et al. Paclitaxel-coated balloon angioplasty versus drug-eluting stent in acute myocardial infarction. JACC: Cardiovasc Interv. 2019; 12: 1691-9.

34. Rittger $\mathrm{H}$, Waliszewski $\mathrm{M}$, Brachmann J, et al. Long-term outcomes after treatment with a paclitaxelcoated balloon versus balloon angioplasty: insights from the PEPCAD-DES study (treatment of drugeluting stent [DES] in-stent restenosis with SeQuent Please paclitaxel-coated percutaneous transluminal coronary angioplasty [PTCA] Catheter). JACC: Cardiovasc Interv. 2015; 8: 1695-700.

35. Candy N, Ng E and Velu R. Paclitaxel-coated balloon reduces target lesion revascularization compared with standard balloon angioplasty. J Vasc Surg. 2017; 65: 558-70.

\section{Tables}

Table 1 Baseline characteristics 


\begin{tabular}{lccc}
\hline N=219 & $\begin{array}{c}\text { CB group } \\
(\mathrm{n}=117)\end{array}$ & $\begin{array}{c}\text { PCB group } \\
(\mathrm{n}=102)\end{array}$ & P value \\
\hline Clinical parameters & $63 \pm 10$ & $64 \pm 11$ & 0.530 \\
Age, years & $95(81.2)$ & $82(80.4)$ & 0.880 \\
Male, n(\%) & $32(27.4)$ & $33(32.4)$ & 0.419 \\
Diabetes, n(\%) & $65(55.6)$ & $52(51.0)$ & 0.498 \\
Hypertension, n(\%) & $28(23.9)$ & $24(23.5)$ & 0.944 \\
Hyperlipidemia, n(\%) & $63(53.9)$ & $58(56.9)$ & 0.654 \\
Smoking, n(\%) & $74(63.3)$ & $66(64.7)$ & 0.823 \\
ACEI/ARB, n(\%) & $84(71.8)$ & $70(68.6)$ & 0.609 \\
ß-blocker, n(\%) & $115(98.3)$ & $100(98.0)$ & 0.890 \\
Statins, n(\%) & $117(100.0)$ & $102(100.0)$ & 1.000 \\
P2Y12 antagonist, n(\%) & $117(100.0)$ & $102(100.0)$ & 1.000 \\
Aspirin, n(\%) & & & \\
Bifurcation site & $46(39.3)$ & $60(58.8)$ & 0.004 \\
LM/LAD/LCX & $61(52.1)$ & $37(36.3)$ & 0.019 \\
LAD/Diagonal & $10(8.6)$ & $5(4.9)$ & 0.287 \\
LCX/OM & & & 0.030 \\
Medina classification & $21(18.0)$ & $27(26.5)$ & \\
1.1.1 & $86(73.5)$ & $58(56.9)$ & \\
1.0.1 & $10(8.6)$ & $17(16.7)$ & \\
0.1.1 & & \\
Pre-procedure & & & \\
Main vessel diameter (mm) & $3.01 \pm 0.35$ & $2.96 \pm 0.43$ & 0.297 \\
Main vessel MLD (mm) & $0.98 \pm 0.38$ & $0.93 \pm 0.38$ & 0.298 \\
Main vessel stenosis (\%) & $67.66 \pm 11.80$ & $68.80 \pm 11.34$ & 0.470 \\
Side branch diameter (mm) & $2.47 \pm 0.38$ & $2.52 \pm 0.35$ & 0.296 \\
Side branch MLD (mm) & $0.59 \pm 0.33$ & $0.62 \pm 0.33$ & 0.474 \\
Side branch stenosis (\%) & $75.95 \pm 13.17$ & $75.37 \pm 12.38$ & 0.737 \\
Post-procedure & & & \\
Main vessel MLD (mm) & $2.51 \pm 0.32$ & $2.46 \pm 0.39$ & 0.408 \\
Main vessel stenosis (\%) & $16.70 \pm 4.58$ & $16.53 \pm 5.16$ & 0.802 \\
Side branch MLD (mm) & $1.98 \pm 0.32$ & $2.06 \pm 0.33$ & 0.079 \\
Side branch stenosis (\%) & $20.05 \pm 5.13$ & $18.59 \pm 6.16$ & 0.057 \\
\hline
\end{tabular}

Data are expressed as the mean \pm SD or $\mathrm{n}(\%)$. ACEI: angiotensin converting enzyme inhibitor; ARB: angiotensin receptor blocker; LM: left main; LAD: left anterior descending; LCX: left circumflex; OM: obtuse marginal; MLD: minimum lumen diameter.

Table 2 Procedure characteristics after the 12-month follow-up

\begin{tabular}{lccc}
\hline N=219 & $\begin{array}{c}\text { CB group } \\
(\mathrm{n}=117)\end{array}$ & $\begin{array}{c}\text { PCB group } \\
(\mathrm{n}=102)\end{array}$ & $P$ value \\
\hline Main vessel MLD (mm) & $2.37 \pm 0.34$ & $2.35 \pm 0.46$ & 0.805 \\
Main vessel LLL (mm) & $0.13 \pm 0.14$ & $0.12 \pm 0.14$ & 0.443 \\
Side branch MLD (mm) & $1.8 \pm 0.43$ & $1.97 \pm 0.36$ & 0.007 \\
Side branch LLL (mm) & $0.19 \pm 0.25$ & $0.11 \pm 0.18$ & 0.024 \\
\hline
\end{tabular}


Data are expressed as the mean \pm SD. MLD: minimum lumen diameter; LLL: late lumen loss.

Table 3 Cardiovascular outcomes during the 12-month follow-up

\begin{tabular}{|c|c|c|c|c|c|c|}
\hline & $\begin{array}{l}\text { CB } \\
\text { group }\end{array}$ & $\begin{array}{l}\text { PCB } \\
\text { group }\end{array}$ & HR(95\% CI) & $P$ value & $\begin{array}{l}\text { Adjusted } \\
\text { HR(95\% CI) }\end{array}$ & $P$ value \\
\hline Restenosis, & $9(7.7)$ & $5(4.9)$ & $0.620(0.208-$ & 0.383 & $0.536(0.176-$ & 0.273 \\
\hline $\begin{array}{l}\mathrm{n}(\%) \\
\mathrm{MACE}, \mathrm{n}(\%)\end{array}$ & $28(23.9)$ & 13(12.8) & $\begin{array}{l}1.850) \\
0.489(0.253-\end{array}$ & 0.028 & $\begin{array}{l}1.634) \\
0.480(0.244-\end{array}$ & 0.033 \\
\hline Angina pectoris, & $23(19.7)$ & $11(10.8)$ & $0.515(0.251-$ & 0.062 & $0.515(0.247-$ & 0.078 \\
\hline $\begin{array}{l}\text { Nonfatal MI, } \\
\text { n(\%) }\end{array}$ & $7(6.0)$ & $3(2.9)$ & $\begin{array}{l}0.485(0.126- \\
1.877)\end{array}$ & 0.283 & $0.453(0.111-$ & 0.270 \\
\hline $\begin{array}{l}\text { Cardiac death, } \\
\text { n(\%) }\end{array}$ & $1(0.9)$ & $1(1.0)$ & $\begin{array}{l}1.142(0.071- \\
18.260)\end{array}$ & 0.925 & $\begin{array}{l}1.089(0.065- \\
18.219)\end{array}$ & 0.953 \\
\hline
\end{tabular}

Results are expressed as n (\%); MACE: major adverse cardiovascular events; MI: myocardial infarction.

Figures 


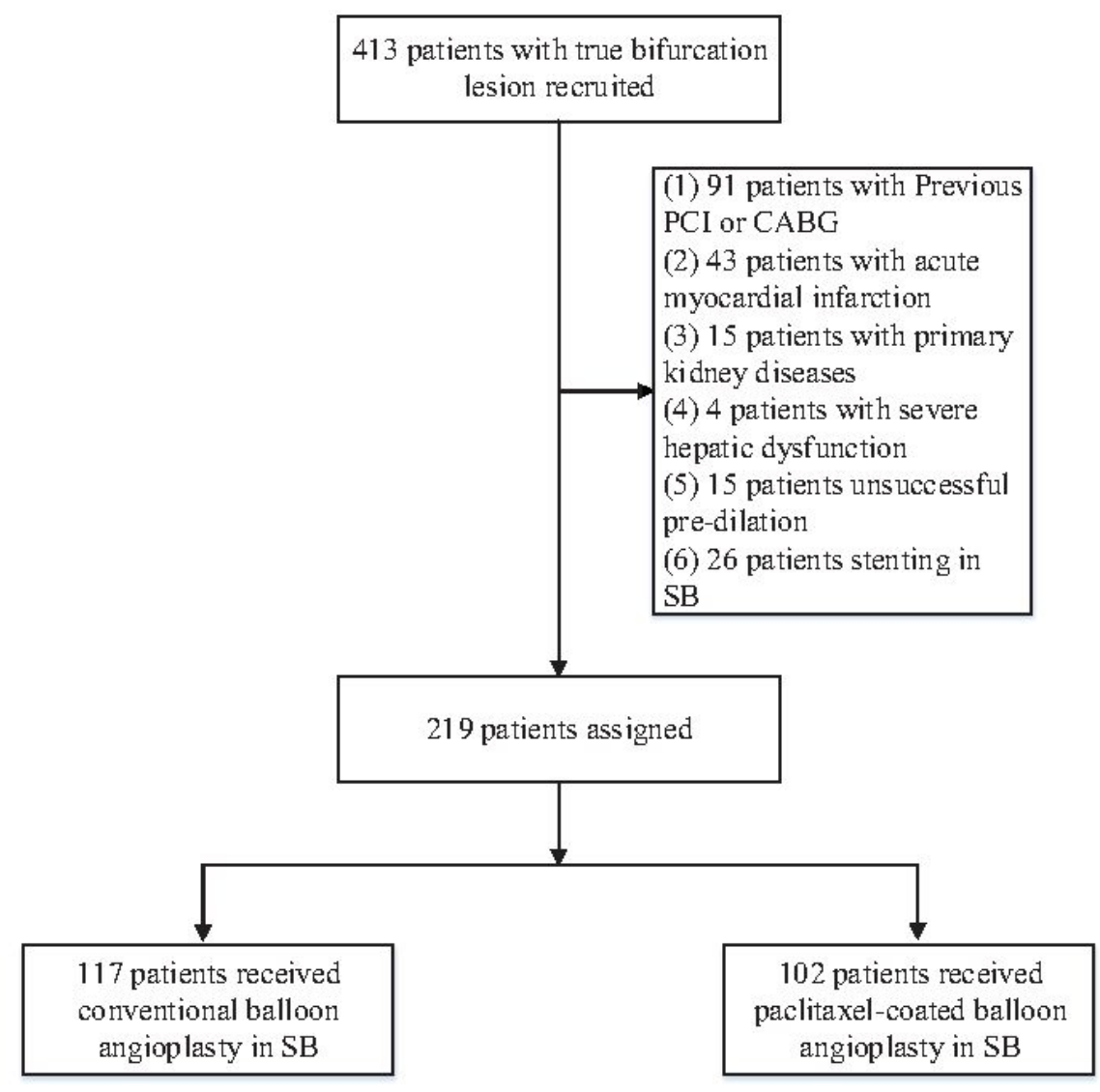

Figure 1

Flow chart 
a

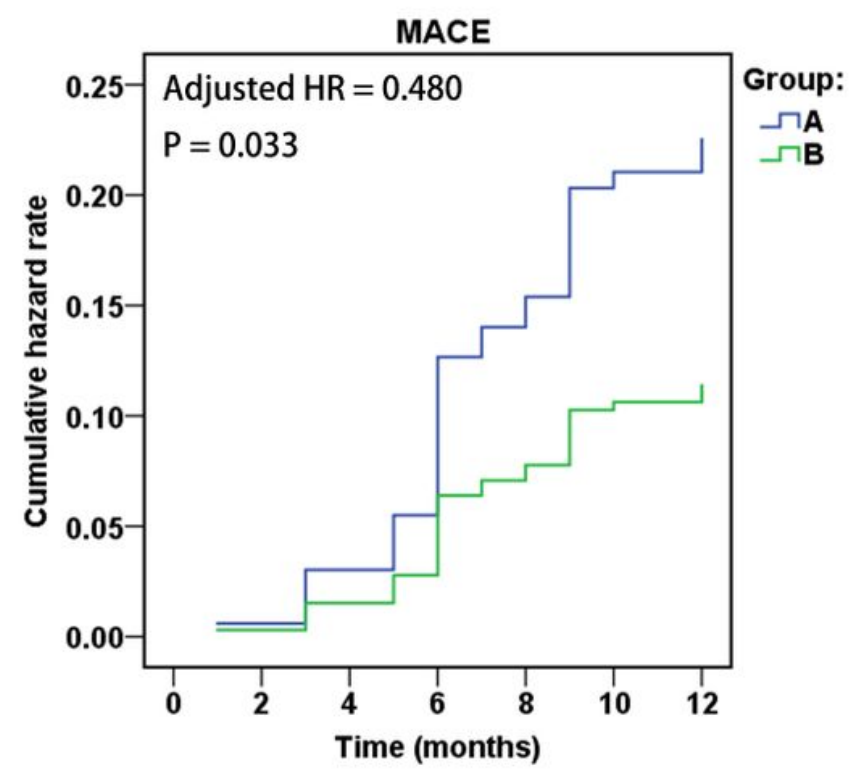

b

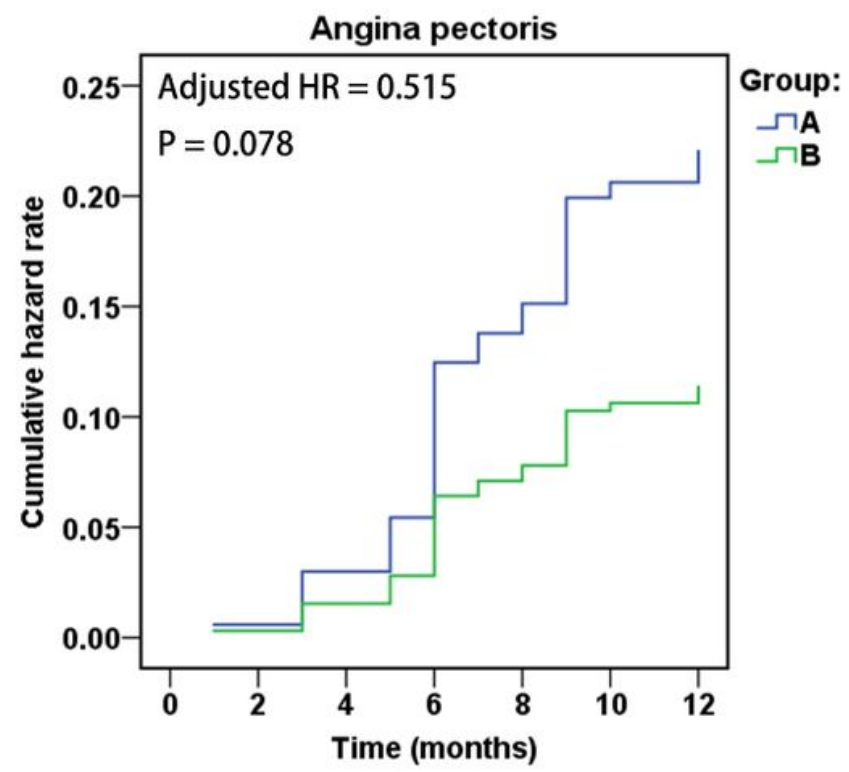

C

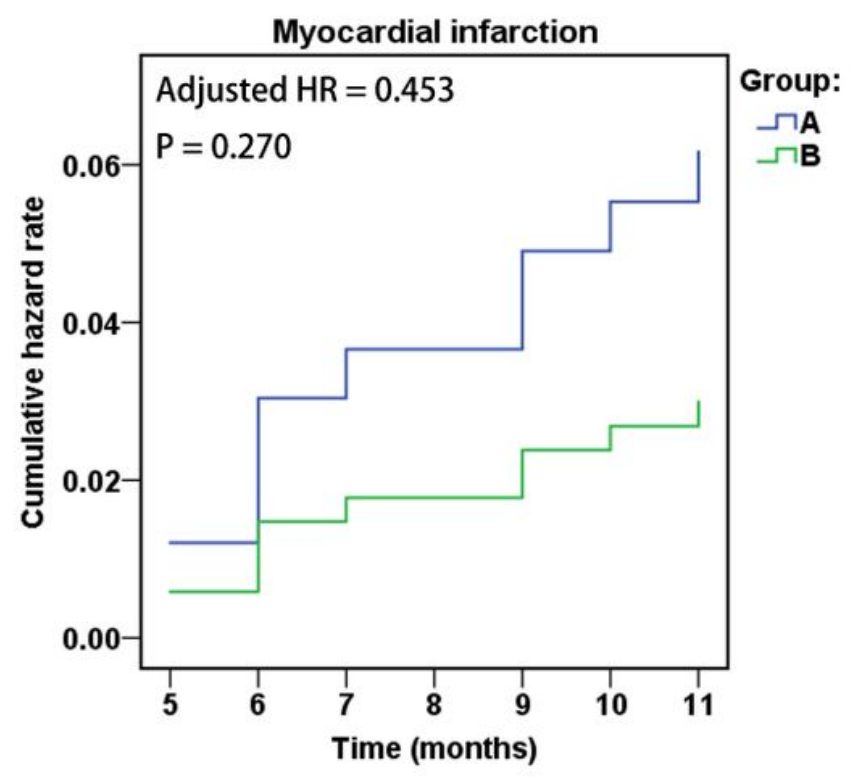

Figure 2

Cumulative risk of cardiovascular events in patients with de novo true bifurcation lesion undergoing $\mathrm{PCl}$ Group A: CB group; Group B: PCB group

\section{Supplementary Files}

This is a list of supplementary files associated with this preprint. Click to download.

- formula.docx 